\title{
IDENTIFICAÇÃO E CARACTERIZAÇÃO DAS ESPÉCIES ARBÓREAS DO CANTEIRO CENTRAL DA UNIVERSIDADE FEDERAL DE LAVRAS/MG
}

\author{
Identification and characterization of the trees species of the \\ central garden of the Universidade Federal of Lavras/MG
}

\begin{abstract}
Patrícia Duarte de Oliveira Paiva ${ }^{1}$, Paulo Roberto Corrêa Landgraf ${ }^{2}$, Tatiana Michlovská Rodrigues ${ }^{3}$, Deborah de Oliveira Pedroso ${ }^{4}$, Ary Teixeira de Oliveira Filho ${ }^{5}$, Manuel Losada Gavilanes ${ }^{6}$, Renato Paiva ${ }^{6}$
\end{abstract}

\begin{abstract}
RESUMO
O campus da Universidade Federal de Lavras possui 508 ha, sendo 117 ha de área construída onde se localizam os departamentos e setores. Nessa área está o canteiro central, que divide as duas principais avenidas da universidade. Essa área é arborizada, porém, não há nenhum mapa com a localização das espécies. Objetivou-se então realizar um inventário dessas árvores, fazendo-se a locação, identificação, quantificação e caracterização delas. A identificação foi feita mediante observação, consulta com outros profissionais, bibliografia especializada e herbário. Foram identificadas 46 espécies arbóreas distribuídas num total de 182 exemplares, pertencentes a 24 famílias. A espécie Callistemon viminalis G.Don ex Loud, popularmente conhecida como escova-de-garrafa, foi a que apresentou maior ocorrência, com 23 indivíduos, seguida do ipê-roxo (Tabebuia impetiginosa (Mart.) Standl.), com 15 indivíduos. Das espécies plantadas, 84\% são nativas. Observou-se uma variedade de espécies locadas cumprindo o objetivo do plantio para uso em aulas práticas e embelezamento.
\end{abstract}

Termos para indexação: Árvores, arborização, árvores nativas.

\section{ABSTRACT}

The campus of the Universidade Federal de Lavras has 508 ha, being 117 of this area ocupped by departments and sections. In this area is located the central garden, which divides the campus in two principal avenues. This area is planted with great number of trees however any map doesn't exist with the location of the species. It was aimed at then to accomplish an inventory of these trees, through doing the lease, identification, quantification and characterization of them. The identification was made through observation, it consults with other professionals, specialized bibliography and herbarium research. There were identified 46 tree species distributed in a total of 182 types, belonging to 24 families. The species Callistemon viminalis G.Don ex Loud, popularly known as "bottle brush" it was the one that presented larger occurrence, with 23 individuals, followed by the ipê-roxo (Tabebeuia impetiginosa (Mart.) Standl.) with 15 individuals. Between the planted species, $84 \%$ are native. We observed a great number of species cultivated in the campus which are used with the objectives to help in practical classes and embellishment.

Index Terms: Tree, native tree.

(Recebido para publicação em 23 de outubro de 2002 e aprovado em 6 de janeiro de 2003)

\section{INTRODUÇÃO}

O verde urbano é fundamental para atenuar os problemas ambientais e sociais que o habitante da cidade enfrenta. Sua importância envolve tanto aspectos paisagísticos, como psicológicos, além de a vegetação possuir importante função nos centros urbanizados no que diz respeito à qualidade ambiental (LIMA 1991).
Segundo Martins Junior (1996), a arborização pode contribuir para a recuperação do ambiente das cidades, tanto nos seus aspectos ecológicos como nos sócio-econômicos. Os parques e áreas verdes devem formar um conjunto natural que resguarde uma beleza cênica à disposição de todos. Milano e Disperati (1995) ressaltam que a vegetação urbana é representada pelas áreas verdes públicas e particulares e pela arborização de ruas e avenidas.

\footnotetext{
1. Professora do Departamento de Agricultura, Universidade Federal de Lavras/UFLA, Caixa Postal 37 - 37200-000 - Lavras, MG. pdolivei@ufla.br 2. Professor, Universidade de Alfenas/UNIFENAS, Alfenas, MG, paulo.landgraf@unifenas.br

3. Doutoranda, UFLA.

4. Engenheira Florestal.

5. Professor do Departamento de Ciências Florestais, UFLA.

6. Professor do Departamento de Biologia, UFLA. gavilane@ufla.br; renpaiva@ufla.br
} 
A degradação das paisagens urbanas está assumindo proporções insustentáveis, no que diz respeito tanto à quantidade como à qualidade. A vegetação, com suas funções ecológicas, econômicas e sociais, pode desempenhar importante papel na melhoria de vida das populações urbanas. De acordo com Milano (1995), a arborização urbana compreende áreas naturais, que apresentem todo e qualquer tipo de vegetação, incluindo desde áreas gramadas até outras com vegetação de porte arbóreo.

Além da melhoria na saúde psíquica do homem, entre os benefícios que esses "conjuntos de árvores" proporcionam, segundo Milano (1995), estão: melhoria microclimática do meio urbano, com a redução das incidências dos raios solares, em outras palavras, sombra; proteção e formação de barreiras contra o vento; redução da temperatura do ar e pela evapotranspiração; ação contra a poluição, já que suas folhas possuem capacidade de absorver e filtrar os gases poluentes, além de reter partículas de poeira do ar; ação acústica e visual, visto que os vegetais são capazes de atenuar e diminuir a reverberação dos ruídos comuns aos centros urbanos, como motores de carros, ônibus e caminhões, buzinas, e outros; benefícios sociais, econômicos e políticos gerados pela valorização das propriedades situadas próximas aos espaços arborizados.

A Universidade Federal de Lavras apresenta uma área total de 508 ha, dos quais 117 ha são de áreas urbanizadas, 207 ha de áreas não urbanizada cultivada, 184 ha de áreas não urbanizadas e não cultivadas. A maior porcentagem de área verde está localizada no canteiro central, que se inicia próximo à Prefeitura do Campus e termina próximo ao Setor de Cafeicultura. O canteiro central possui uma área aproximada de 2,3 ha, arborizado com espécies nativas e exóticas, sendo a disposição de plantio de forma aleatória. Há necessidade de se conhecer essas espécies, que devem e podem desempenhar suas funções no que tange à ecologia, a questões ambientais, ao lazer, à integração, à estética e à pesquisa, isto é, tomar conhecimento da influência que a vegetação exerce nos parâmetros climáticos de diversas áreas e seus arredores, reduzindo a radiação, a temperatura, gerando a elevação da umidade e reduzindo a velocidade do vento.

Com o presente trabalho objetivou-se fazer a identificação, a locação, a quantificação e caracterização das espécies arbóreas localizadas no canteiro central do Campus da Universidade Federal de Lavras (UFLA), fornecendo o conhecimento básico para o uso em pesquisa, coleta de sementes e aulas práticas.

\section{MATERIAL E MÉTODOS}

O presente estudo foi realizado no canteiro central do Campus da Universidade Federal de Lavras, Lavras/MG. A cidade está localizada a $21^{\circ} 14 ' 30$ " latitude S e $45^{\circ} 00^{\prime} 10^{\prime}$ ' longitude W, e altitude de $918 \mathrm{~m}$ acima do nível do mar. O clima, de acordo com Koppen, é do tipo $C w b$ : mesotérmico, de verões brandos e chuvosos. As temperaturas médias do mês mais frio e do mais quente são inferiores a $18^{\circ}$ e $22^{\circ}$, respectivamente, com temperatura média anual $19,3^{\circ} \mathrm{C}$. Os índices pluviométricos anuais situam-se em torno de $1.411 \mathrm{~mm}$, sendo o período mais chuvoso de dezembro a março e o período de estiagem coincidente com os meses de junho, julho e agosto.

De acordo com a Prefeitura do campus da UFLA, a área total da universidade abrange 508 ha, e desses, $23 \%$ representam área urbanizada; $41 \%$, área não urbanizada, mas cultivada; e 36\%, área não urbanizada e não cultivada. $O$ canteiro central representa $1 \%$ da área do Campus Universitário.

A planta com a locação do canteiro central foi adquirida na Prefeitura do Campus da UFLA. Nessa planta, foram locadas e identificadas todas as espécies arbóreas existentes na área. Utilizou-se para essa locação na planta o programa AUTOCAD R14. As árvores existentes foram identificadas com seu nome vulgar, nome científico e família, além de outras características para compor o relatório final.

Realizou-se um levantamento das árvores, identificando as espécies, realizando medições (em relação ao meio-fio) para posterior locação na planta e contando-se o número de exemplares de cada indivíduo.

\section{RESULTADOS E DISCUSSÃO}

No levantamento, identificaram-se 46 espécies arbóreas plantadas no canteiro central da UFLA; dessas, $84 \%$ são nativas e $16 \%$, exóticas. Essas espécies foram plantadas de forma aleatória, com finalidade de embelezamento do local e também com o objetivo da utilização dessas plantas para aulas práticas.

A espécie Callistemon viminalis G.Don ex Loud, popularmente conhecida como escova-degarrafa, foi a que apresentou maior ocorrência, com 23 indivíduos, seguida do ipê-roxo (Tabebuia impetiginosa (Mart.) Standl.), com 15 indivíduos. Foram encontradas espécies raras como o mogmo 
(Swietenia macrophylla King), com 8 exemplares. Algumas espécies frutíferas, como goiaba, pitanga, jabuticaba e araçá foram identificadas e possivelmente plantadas para a alimentação de pássaros existentes no Campus.
Na Tabela 1 são apresentados os nomes comuns, científico, família, origem, porte, tipo de folha, época de florescimento e a quantidade das espécies arbóreas localizadas no canteiro central da Universidade de Lavras.

TABELA 1 - Espécies arbóreas presentes no canteiro central do Campus da Universidade Federal de Lavras UFLA/2001.

\begin{tabular}{|c|c|c|c|c|c|c|c|c|}
\hline \multirow{2}{*}{$\begin{array}{l}\text { Nome } \\
\text { Comum }\end{array}$} & \multirow{2}{*}{ Nome Científico } & \multirow{2}{*}{ Família } & \multirow{2}{*}{ Origem } & \multirow{2}{*}{ Porte } & \multirow{2}{*}{ Folha } & \multicolumn{2}{|c|}{ Florescimento } & \multirow{2}{*}{$\begin{array}{l}\mathbf{N}^{\mathbf{o}} \\
\text { sp. }\end{array}$} \\
\hline & & & & & & Cor & Época & \\
\hline Acácia & Acacia mangium Willd. & Fabaceae Mimosoideae & Austrália & 4 a $6 \mathrm{~m}$ & $\begin{array}{l}\text { Semicaduc } \\
\text { a }\end{array}$ & Amarela & jul-set & 2 \\
\hline $\begin{array}{l}\text { Angico - } \\
\text { cangalha }\end{array}$ & $\begin{array}{l}\text { Peltophorum dubium } \\
\text { (Sprengel) Taub. }\end{array}$ & Fabaceae Caesalpinioideae & Brasil & $15 \mathrm{~m}$ & Caduca & Amarela & dez-fev & 3 \\
\hline Angiico & $\begin{array}{l}\text { Leucochloron incuriale } \\
\text { (Vell.) Barneby \& Grimes }\end{array}$ & Fabaceae Mimosoideae & Brasil & $18 \mathrm{~m}$ & $\begin{array}{l}\text { Semicaduc } \\
\text { a }\end{array}$ & Creme & set-jan & 1 \\
\hline Araçá & $\begin{array}{l}\text { Psidium cattleyanum } \\
\text { Weinw }\end{array}$ & Myrtaceae & Brasil & $15 \mathrm{~m}$ & Perene & Branca & jun-dez & 5 \\
\hline $\begin{array}{l}\text { Azeitona do } \\
\text { Ceilão }\end{array}$ & Elaeocarpus serratus L. & Elaeocapaceae & $\begin{array}{l}\text { Índia, } \\
\text { Ceilão }\end{array}$ & - & Perene & Branca & jun-out & 1 \\
\hline $\begin{array}{l}\text { Pinheiro-do- } \\
\text { Paraná }\end{array}$ & $\begin{array}{l}\text { Araucaria angustifolia } \\
\text { (Bert.) Kuntze. }\end{array}$ & Araucariaceae & Brasil & $50 \mathrm{~m}$ & Perene & $\begin{array}{l}\text { Sem flor } \\
\text { verdadeira }\end{array}$ & - & 1 \\
\hline $\begin{array}{l}\text { Aroeira-salsa, } \\
\text { chorão- } \\
\text { mexicano }\end{array}$ & Schinus molle L.. & Anacardiaceae & $\begin{array}{l}\text { América } \\
\text { Central }\end{array}$ & $8 \mathrm{~m}$ & Perene & Esverdeada & ago-nov & 2 \\
\hline Barriguda & $\begin{array}{l}\text { Ceiba erianthos (Cav.) K. } \\
\text { Schum. }\end{array}$ & Malvaceae (Bombacaceae) & Brasil & $30 \mathrm{~m}$ & Caduca & Rosa & abr-jun & 1 \\
\hline $\begin{array}{l}\text { Bauínia, } \\
\text { árvore-orquídea }\end{array}$ & Bauhinia variegata $\mathrm{L}$. & Fabaceae Caesalpinioideae & China & $7 \mathrm{~m}$ & $\begin{array}{l}\text { Semicaduc } \\
\text { a }\end{array}$ & $\begin{array}{l}\text { Lilás, } \\
\text { branca }\end{array}$ & jul-out & 13 \\
\hline Cabeludinha & $\begin{array}{l}\text { Paramyrciaria glomerata } \\
\text { (O.Berg) Sobral }\end{array}$ & Mytaceae & Brasil & $4 \mathrm{~m}$ & Perene & Branca & jul-out & 2 \\
\hline Calabura & Muntinginia calabura $\mathrm{L}$. & Muntingiaceae & $\begin{array}{l}\text { América } \\
\text { Central }\end{array}$ & $12 \mathrm{~m}$ & Perene & Branca & ago-dez & 2 \\
\hline Canafístula & $\begin{array}{l}\text { Cassia ferruginea Schrad } \\
\text { ex DC }\end{array}$ & Fabaceae Caesalpinioideae & Brasil & $20 \mathrm{~m}$ & Caduca & Amarela & dez-fev & 3 \\
\hline
\end{tabular}


TABELA 1 - Continuação...

\begin{tabular}{|c|c|c|c|c|c|c|c|c|}
\hline \multirow{2}{*}{$\begin{array}{l}\text { Nome } \\
\text { Comum }\end{array}$} & \multirow{2}{*}{ Nome Científico } & \multirow{2}{*}{ Família } & \multirow{2}{*}{ Origem } & \multirow{2}{*}{ Porte } & \multirow{2}{*}{ Folha } & \multicolumn{2}{|c|}{ Florescimento } & \multirow{2}{*}{$\begin{array}{l}\mathbf{N}^{\circ} \\
\text { sp. }\end{array}$} \\
\hline & & & & & & Cor & Época & \\
\hline $\begin{array}{l}\text { Cerejeira-do- } \\
\text { Japão }\end{array}$ & Prunus serrulata Lindl. & Rosaceae & Japão & $15 \mathrm{~m}$ & Caduca & Rosa & jul-set & 1 \\
\hline Embaúba & Cecropia sp. & Cecropiaceae & Bolívia & $10 \mathrm{~m}$ & Perene & Branca & set-out & 1 \\
\hline $\begin{array}{l}\text { Escova-de- } \\
\text { garrafa }\end{array}$ & $\begin{array}{l}\text { Callistemon viminalis } \\
\text { G.Don ex Land }\end{array}$ & Myrtaceae & Austrália & $10 \mathrm{~m}$ & Perene & Vermelha & out-mar & 23 \\
\hline Ficus & Ficus lyrata Warb. & Moraceae & Ásia & $10 \mathrm{~m}$ & Perene & $\begin{array}{l}\text { Flores em } \\
\text { sicônio }\end{array}$ & Variada & 2 \\
\hline Flamboyant & Delonix regia Raf. & $\begin{array}{l}\text { Fabaceae } \\
\text { Caesalpinioideae }\end{array}$ & Madagascar & $8 \mathrm{~m}$ & Caduca & $\begin{array}{c}\text { Amarela/ } \\
\text { laranja/ } \\
\text { vermelha }\end{array}$ & out-dez & 2 \\
\hline Grevilea & $\begin{array}{l}\text { Grevillea robusta A. } \\
\text { Cunn. }\end{array}$ & Proteaceae & Austrália & $25 \mathrm{~m}$ & Caduca & $\begin{array}{c}\text { Amarela/ } \\
\text { laranja }\end{array}$ & ago-dez & 1 \\
\hline Ipê-amarelo & $\begin{array}{l}\text { Tabebuia serratifolia } \\
\text { Nichols }\end{array}$ & Bignoniaceae & Brasil & $25 \mathrm{~m}$ & Caduca & Amarela & jul-out & 5 \\
\hline Ipê-branco & $\begin{array}{l}\text { Tabebuia roseo-alba } \\
\text { (Ridl.) Sand.w }\end{array}$ & Bignoniaceae & Brasil & $18 \mathrm{~m}$ & Caduca & Branca/ rósea & set-out. & 6 \\
\hline $\begin{array}{l}\text { Ipê-amarelo- } \\
\text { do-cerrado }\end{array}$ & $\begin{array}{l}\text { Tabebuia ochracea } \\
\text { (Cham.) Standl. }\end{array}$ & Bignoniaceae & Brasil & $12 \mathrm{~m}$ & Caduca & Amarela & jul-set & 5 \\
\hline Ipê-roxo & $\begin{array}{l}\text { Tabebuia impetiginosa } \\
\text { (Mart.) Standley }\end{array}$ & Bignoniaceae & Brasil & $25 \mathrm{~m}$ & Caduca & Roxa/rosa-forte & ago-set & 15 \\
\hline Jabuticabeira & $\begin{array}{l}\text { Myrciaria cauliflora } \mathrm{O} . \\
\text { Berg. }\end{array}$ & Myrtaceae & Brasil & $15 \mathrm{~m}$ & Perene & Branca & out-nov & 1 \\
\hline $\begin{array}{l}\text { Jacarandá-da- } \\
\text { baía }\end{array}$ & $\begin{array}{l}\text { Dalbergia nigra (Vell.) } \\
\text { Allem ex Benth }\end{array}$ & Fabaceae Faboideae & Brasil & $25 \mathrm{~m}$ & Semicaduca & Branca & jan-fev & 7 \\
\hline $\begin{array}{l}\text { Jacarandá- } \\
\text { banana }\end{array}$ & $\begin{array}{l}\text { Swartzia langsdorffii } \\
\text { Raddi }\end{array}$ & Fabaceae Faboideae & Brasil & $20 \mathrm{~m}$ & Perene & Branca & set-jan & 4 \\
\hline $\begin{array}{l}\text { Jacarandá-de- } \\
\text { minas }\end{array}$ & $\begin{array}{l}\text { Jacaranda cuspidifolia } \\
\text { Mart. }\end{array}$ & Bignoniaceae & Brasil & $15 \mathrm{~m}$ & Caduca & Lilás & set-out & 3 \\
\hline $\begin{array}{l}\text { Jacarandá- } \\
\text { mimoso } \\
\end{array}$ & $\begin{array}{l}\text { Jacaranda mimosifolia D. } \\
\text { Don. }\end{array}$ & Bignoniaceae & Argentina & $12 \mathrm{~m}$ & Caduca & Lilás & set-dez & 5 \\
\hline $\begin{array}{l}\text { Jambolão, } \\
\text { jamelão }\end{array}$ & $\begin{array}{l}\text { Syzygium cumini (L.) } \\
\text { Skeels }\end{array}$ & Myrtaceae & Índia & $15 \mathrm{~m}$ & Ásia & Branca & jan & 3 \\
\hline Jatobá & Hymenaea courbaril L. & $\begin{array}{l}\text { Fabaceae } \\
\text { Caesalpinioideae }\end{array}$ & Brasil & $30 \mathrm{~m}$ & Semicaduca & Creme & jan-mar & 3 \\
\hline $\begin{array}{l}\text { Jequitibá- } \\
\text { branco }\end{array}$ & $\begin{array}{l}\text { Cariniana estrellensis } \\
\text { (Raddi) Kuntze }\end{array}$ & Lecythidaceae & Brasil & $45 \mathrm{~m}$ & Semicaduca & Branca & set-nov & 1 \\
\hline $\begin{array}{l}\text { Magnólia- } \\
\text { amarela }\end{array}$ & Michelia champaca $\mathrm{L}$. & Magnoliaceae & Índia & $8 \mathrm{~m}$ & Semicaduca & Amarela & set-jan & 3 \\
\hline Manga & Mangifera indica $\mathrm{L}$. & Anacardiaceae & Índia & $15 \mathrm{~m}$ & Perene & Creme & set-dez & 3 \\
\hline Mirindiba-rosa & $\begin{array}{l}\text { Lafoensia glyptocarpa } \\
\text { Koehne }\end{array}$ & Lythraceae & Brasil & $20 \mathrm{~m}$ & Perene & Branca/rosa & jul-set & 4 \\
\hline Mogno & $\begin{array}{l}\text { Swietenia macrophylla } \\
\text { King }\end{array}$ & Meliaceae & Brasil & $\begin{array}{c}25-30 \\
\mathrm{~m}\end{array}$ & Caduca & Creme & nov-dez & 8 \\
\hline Murici & $\begin{array}{l}\text { Byrsonima crassifolia } \\
\text { Stered }\end{array}$ & Malpighiaceae & Brasil & $10 \mathrm{~m}$ & Perene & Amarela & nov-mar & 4 \\
\hline Oiti & Licania tomentosa Fritsch & Rosaceae & Brasil & $10 \mathrm{~m}$ & Perene & Branca & jul-set & 3 \\
\hline Óleo-copaíba & $\begin{array}{l}\text { Copaifera langsdorffii } \\
\text { Desf. }\end{array}$ & $\begin{array}{l}\text { Fabaceae } \\
\text { Caesalpinioideae }\end{array}$ & Brasil & $25 \mathrm{~m}$ & Semicaduca & Creme & nov-fev & 2 \\
\hline Ormósia & Ormosia fastigiata Tul. & Fabaceae Faboideae & Brasil & $20 \mathrm{~m}$ & Perene & Lilás & out-dez & 2 \\
\hline Pau-brasil & Caesalpinia echinata Lam. & $\begin{array}{l}\text { Fabaceae } \\
\text { Caesalpinioideae }\end{array}$ & Brasil & $25 \mathrm{~m}$ & Semicaduca & Amarela & out-dez & 1 \\
\hline
\end{tabular}


TABELA 1 - Continuação...

\begin{tabular}{|c|c|c|c|c|c|c|c|c|}
\hline \multirow{2}{*}{$\begin{array}{l}\text { Nome } \\
\text { Comum }\end{array}$} & \multirow{2}{*}{ Nome Científico } & \multirow{2}{*}{ Família } & \multirow{2}{*}{ Origem } & \multirow{2}{*}{ Porte } & \multirow{2}{*}{ Folha } & \multicolumn{2}{|c|}{ Florescimento } & \multirow{2}{*}{$\begin{array}{l}\mathrm{N}^{\mathbf{o}} \\
\text { sp. }\end{array}$} \\
\hline & & & & & & Cor & Época & \\
\hline Pau-ferro & Caesalpinia ferrea Mart & Fabaceae Caesalpinioideae & Brasil & $20 \mathrm{~m}$ & $\begin{array}{l}\text { Semicaduc } \\
\text { a }\end{array}$ & Amarela & jan-mar & 5 \\
\hline Pau-jangada & $\begin{array}{l}\text { Ochroma pyramidale } \\
\text { (Cav. Ex Lam) Urban }\end{array}$ & Malvaceae (Bombacaceae) & Brasil & $15 \mathrm{~m}$ & $\begin{array}{l}\text { Semicaduc } \\
\mathrm{a}\end{array}$ & Creme & maio-ago & 9 \\
\hline Pinus-elioti & Pinus elliottii Engelm. & Pinaceae & EUA & $25 \mathrm{~m}$ & Perene & $\begin{array}{c}\text { Sem flor } \\
\text { verdadeira }\end{array}$ & - & 1 \\
\hline Pinus-pátula & $\begin{array}{l}\text { Pinus patula Schiede ex. } \\
\text { Schltdl \& Cham }\end{array}$ & Pinaceae & México & $20 \mathrm{~m}$ & Perene & $\begin{array}{c}\text { Sem flor } \\
\text { verdadeira }\end{array}$ & - & 5 \\
\hline Pitangueira & Eugenia uniflora $\mathrm{L}$. & Myrtaceae & Brasil & $6 \mathrm{~m}$ & $\begin{array}{l}\text { Semicaduc } \\
\mathrm{a} \\
\end{array}$ & Branca & out-jan & 4 \\
\hline Quaresmeira & $\begin{array}{l}\text { Tibouchina granulosa } \\
\text { Cogn. }\end{array}$ & Melastomataceae & Brasil & $10 \mathrm{~m}$ & Perene & Rosa/roxa & jan-abr & 4 \\
\hline Saboneteira & Sapindus saponaria $\mathrm{L}$. & Sapindaceae & Brasil & $10 \mathrm{~m}$ & $\begin{array}{c}\text { Semicaduc } \\
\mathrm{a} \\
\end{array}$ & Creme & & 1 \\
\hline Sibipiruna & $\begin{array}{l}\text { Caesalpinia pluviosa DC. } \\
\text { (=C. peltophoroides } \\
\text { Benth.) }\end{array}$ & Fabaceae Caesalpinioideae & Brasil & $20 \mathrm{~m}$ & Perene & Amarela & jan-abr & 2 \\
\hline Sombreiro & $\begin{array}{l}\text { Clitoria fairchildiana } \\
\text { Howard }\end{array}$ & Fabaceae Faboideae & Brasil & $15 \mathrm{~m}$ & Caduca & Arroxeada & dez-maio & 1 \\
\hline Tamarindo & Tamarindus indica $\mathrm{L}$. & Fabaceae Faboideae & India & $15 \mathrm{~m}$ & $\begin{array}{l}\text { Semicaduc } \\
\text { a }\end{array}$ & Creme & set-out & 1 \\
\hline Uvaia & $\begin{array}{l}\text { Eugenia pyriformis } \\
\text { Cambess. }\end{array}$ & Myrtaceae & Brasil & $4 \mathrm{~m}$ & Perene & Creme & set-dez & 1 \\
\hline
\end{tabular}

\section{CONCLUSÕES}

Após a realização deste trabalho, pode-se concluir que existem 46 espécies de árvores plantadas no canteiro central da UFLA; essas arvores foram plantadas aleatoriamente e estão cumprindo o objetivo do plantio para uso em aulas práticas e embelezamento. O levantamento, quantificação, locação e identificação dessas espécies facilitará o uso em aula prática, pesquisa, coleta de sementes e proporcionará também o conhecimento das espécies existentes na área.

\section{REFERÊNCIAS BIBLIOGRÁFICAS}

LIMA, S. T. de. Verde urbano: uma questão de qualidade ambiental. In: ENCONTRO NACIONAL
DE ESTUDOS SOBRE O MEIO AMBIENTE, 3., 1991, Londrina. Anais... Londrina: [s.n.], 1991. p. 517-529.

MARTINS JÚNIOR, O. P. Uma cidade ecologicamente correta. Goiânia: A.B., 1996.

MILANO, M. S. Arborização urbana. Curitiba: UFPr, 1995. Apostila.

MILANO, M. S.; DISPERATI, A. A. Análise da quantidade e distribuição das áreas verdes no município de Curitiba - PR. Curitiba: UFPr, 1995. Apostila do curso sobre arborização urbana Universidade Livre do meio Ambiente. 